\title{
Classical liquid in a box
}

A simulation of a fluid of interacting particles in a box much narrower in one dimension than the other two reveals a curious bimodal density distribution along the short dimension - and provides a telling example of Poisson statistics.

THAT the perennial question of whether fashions for problems of a particular kind precede or follow the arrival of means for their solution will never be settled is nicely illustrated by the recent interest in smallish clusters of atoms or molecules. The recognition of small clusters in atomic or molecular beams almost coincided with the point at which the techniques of molecular physics could be turned in that direction with some chance of success.

That, no doubt, is one reason why the problems of molecular systems whose dimensions are essentially atomic are now all the rage. But that does not diminish their high interest, which is that of throwing light on the transition between the properties of a collection of isolated molecules and a bulk aggregate thereof. But now there is a model calculation of a system of this kind, essentially a bulk liquid consisting of classical atoms, in which only one of the three physical dimensions is of an atomic scale. Its outcome is full of surprise.

The problem, definable theoretically if unattainable in the machine-shop, is that of a liquid enclosed between infinite parallel plates which are separated from each other by only three atomic diameters. It is the problem of a fluid in a flattened capillary tube. There are obvious connections with that of a thin layer of fluid adsorbed on the surface of a solid as a two-dimensional gas (but the constraints are greater), as well as with the behaviour of a fluid in a porous structure (but "micropore" would be a better word).

J. G. Powles, A. Dornford-Smith and W. A. B. Evans from the University of Kent at Canterbury (England), who have a reputation in the solution of static versions of problems such as this, now say that their interest is in the dynamics of atomic motion in such a system (Phys. Rev. Lett. 66, 1177-1180; 4 March 1991).

Describing the problem is easier than solving it. Pairs of atoms are supposed to interact by means of a Lennard-Jones potential - the standard combination of an attractive force varying with the sixth power of the inverse separation and a repulsive force varying similarly with the twelfth power (which dominates at short distances). The 'walls', simulated as an external potential applicable to all atoms between them, repel all atoms according to the ninth power of their distance, thus preventing what might be called condensation on the surface.

The bare bones of static equilibrium are easily guessed at. If the fluid were a perfect gas, so that the mutual interaction of pairs of molecules vanishes, but if the repulsion of the walls persists, there will be a smooth distribution of molecules within the slab of space between the walls, with maximum density midway between them. Increasing temperature (or giving the atoms kinetic energy) makes the distribution flatter. But what happens if the mutual interaction is switched on? Then there is a relative concentration of molecules in two layers on either side of the midpoint (which is only, in this model, 1.5 atomic diameters from each wall).

Handwaving will get one almost there. Atoms are repelled both by the walls and by those of their own number lying closer than just over one atomic diameter. (The energy minimum of the Lennard-Jones potential is at 1.12 times the number taken to be the diameter.) So a density distribution in which atoms were crowded near the midpoint would be unstable relative to a bimodal distribution, but less so at increased temperature.

In practice, these properties are arrived at by numerical calculation. Powles, DornfordSmith and Evans put 1,020 atoms in a simulated box and follow what happens to them. As it happens, the two long dimensions of the box turn out to be just over 23 atomic diameters - far from infinite, but probably sufficient for the purposes. It turns out that there is little of interest to say about the motion of atoms parallel with the confining walls; even if one of them should embark from a place where the density is a maximum, only rarely will it thread its way through the relatively dense gas at that exact distance from one wall. So classical diffusion is a sufficient picture of what happens.

The fun comes in the direction perpendicular to the wall. What emerges from individual trajectories is that atoms spend the vast majority of the time at or near one or other of the two density maxima between the walls, transferring from one extremum to the other at high speed. At any time, only about ten per cent of the atoms will be in intermediate positions; in other words, the transition time is about a tenth of the average time spent at one of the extrema.

So much has become apparent largely because the numerical calculations consist of iterations of the state of the system at intervals of time that are extremely short compared with the physical characteristics of the system. The basic time-step used, for example, is equivalent to one twentieth of the velocity auto-correlation time, which is an inverse measure of the time over which velocities persist unchanged. A coarser timestep might well have obscured this fine detail.

In itself, this says little for the behaviour of real fluids tightly constrained by external boundaries except that they are unlikely to behave as fluids in the directions in which they are constrained, whatever their properties in perpendicular directions. On the face of things, the transverse behaviour of the fluid involving the hopping of atoms from one favoured position to another may be a sign that the fluid behaves as a liquid in that direction and as a gas in the two other directions.

It would be interesting to see what would happen to this model (as to other models) if the basic parameters were different, and in particular if they were such as to make condensation probable and recognizable (which is not that simple in such a narrow slab of fluid). Whether it would be possible to extend the calculations to atoms with directional preferences, as might be expected of water molecules, is more problematical. Already, the authors note that each time-step of their calculation takes 1.8 seconds of time on an array of 17 parallel-wired transputers - the British-made microprocessors that come nearest to being computers on a single chip. That seems to imply a few days for each simulation.

But Powles, Dornford-Smith and Evans seem most of all intrigued by having stumbled on a neat way of testing whether experimental data fit Poisson statistics. Modishly, they refer to an article dating from 1910 in which Rutherford, Geiger and Bateman regret the difficulty of demonstrating the Poisson nature of radioactive decay by accumulating data about the intervals of time between successive decay-events ( $P$ hil. Mag. 20,698; 1910), chiefly for lack of datahandling techniques.

The case of the constrained fluid is more easily handled. If an event is the crossing of an atom from one extremum to the other, the intervals, $t$, between events are readily calculated and recorded, from which it is possible to work out all possible quantities such as $\left\langle t^{n}\right\rangle$, where $n$ is an integer and the anglebrackets mean averages. But the outstanding characteristic of a Poisson distribution is that $\left\langle t^{n}\right\rangle /\langle t\rangle^{n}=n$ !, whatever the particular parameters defining the distribution. So the constrained fluid is christened a "liquid Poisson oscillator". The name is inconsequential, but the model is not.

John Maddox 